\author{
B. Elma $^{1}$, E. Misirli ${ }^{*}$ iD \\ Ege University, İzmir, Turkey \\ e-mail: berfin-elma@hotmail.com
}

\title{
On the physical behaviours of the conformable fractional modified Camassa - Holm equation using two efficient methods
}

\begin{abstract}
In recent years, many authors have researched about fractional partial differential equations. Physical phenomena, which arise in engineering and applied science, can be defined more accurately by using FPDEs. Thus, obtaining exact solutions of the FPDEs equations have become more important to understand physical problems. In this article, we have reached the new traveling wave solutions of the conformable fractional modified Camassa - Holm equation via two efficient methods such as first integral method and the functional variable method. The wave transformation and conformable fractional derivative have been used to convert FPDE to the ordinary differential equation. The Camassa - Holm equation is physical model of shallow water waves with non-hydrostatic pressure. Thanks to these powerful methods, some comparisons, such as type of solutions and physical behaviours, have been made. Additionally, mathematica program have been used with the aim of checking of solutions. Investigating results of the fractional differential equations can help understanding complex phenomena in applied mathematics and physics.

Key words: Fractional modified Camassa-Holm equation; traveling wave solutions; conformable derivative; the first integral method; the functional variable method.
\end{abstract}

\section{Introduction}

Fractional derivative models can describe many complex problems in physics and engineering more correctly. Recently, fractional derivatives have been used on applications in many area such as signal processing, nonlinear optics, water wave modeling, control theory and etc [1-3]. There are various derivative definitions such as the Riemann Liouville derivative [3], the Caputo derivative [4], Jumarie's modified Riemann - Liouville derivative [5] and the Atangana - Baleanu derivative [6] in literature.

Numerous methods have been applied to obtain different solutions of fractional partial differential equations. Some of them are; the functional variable method [7], the extended tanh function method [8], the first integral method [9], the extended direct algebraic method [10], the modified simple equation method [11], the modified trial equation method [12], the $\left(\boldsymbol{G}^{\prime} / \boldsymbol{G}\right)$ - expansion method [13], the extended trial equation method [14], the kudryashov method [15] and so on [16-25].

In this article, first integral method [26] and functional variable method [27] have been applied to reach the exact solutions of the conformable fractional modified Camassa - Holm equation arising in fluid dynamics. The time-fractional modified Camassa-Holm equation of the following form [28]:

$$
\begin{gathered}
D_{t}^{\alpha} u+2 \sigma u_{x}-u_{x x t}+\beta u^{2} u_{x}= \\
=0 \sigma \in R, \text { and } 0<\alpha \leq 1
\end{gathered}
$$

$D_{t}^{\alpha} u$ is express of the conformable fractional derivative of $u$ with respect to $t$ of order $\alpha$ and $\sigma, \beta$ are non-zero constants.

The rest of article is given as follows: Some definitions and properties of conformable derivative are defined in section 2. In section 3, the proposed methods are applied to the fractional modified Camassa-Holm equation for obtaining some new exact solutions in section 4. In section 5 , a discussion of the results is clarified. In final section, includes a conclusion which contains all outputs in this article.

\section{Conformable Fractional Derivative}

Khalil et al. defined the below theorem for the fractional derivatives [29]:

Let $h:[0, \infty) \rightarrow R$ be a function and its fractional conformable derivative of $h$ order $\alpha$ is,

$$
D_{t}^{\alpha}(h)(x)=\lim _{\varepsilon \rightarrow 0} \frac{h\left(x+\varepsilon x^{1-\alpha}\right)-h(x)}{\varepsilon},
$$

$\alpha \epsilon(0,1) \forall x>0$. Some of important properties of conformable derivative as follows:

Suppose $\alpha E(0,1]$ and $g, h$ be $\alpha$-differentiable at a point $t>0$. Then, 
$T_{\alpha}(a g+b h)=a T_{\alpha}(g)+b T_{\alpha}(h) \forall a, b \in R$.

$T_{\alpha}\left(k^{p}\right)=p k^{p-1} \forall p € R$.

$T_{\alpha}(\beta)=0$, for all constant functions $g(t)=\beta$.

$T_{\alpha}(g h)=g T_{\alpha}(h)+h T_{\alpha}(g)$.

$T_{\alpha}\left(\frac{g}{h}\right)=\frac{h T_{\alpha}(g)-g T_{\alpha}(h)}{g^{2}}$.

If $g$ is differentiable, $T_{\alpha}(g)(t)=t^{1-\alpha} \frac{d g}{d t}(t)$.

\section{First Integral Method}

Introducing the wave transformation:

$$
u(x, t)=U(\xi), \xi=x-k \frac{t^{\alpha}}{\Gamma(1+\alpha)}
$$

Eqn. (1) reduce the form of eqn.(4) an ODE:

$$
k U^{\prime \prime}(\xi)+(2 \sigma-k) U(\xi)+\frac{\beta}{3} U^{3}(\xi)=0
$$

and then we can write two dimensional autonomous system

$$
\begin{gathered}
\frac{d X}{d \xi}=Y(\xi) \\
\frac{d Y}{d \xi}=\frac{k-2 \sigma}{k} X(\xi)-\frac{\beta}{3 k} X^{3}(\xi)
\end{gathered}
$$

According to first integral method, $\mathrm{X}$ and $\mathrm{Y}$ be a non-trivial solutions of the eqn.(5). Also, irreducible polynomial $Q(X, Y)=\sum_{i=0}^{m} a_{i}(X) Y^{\dot{I}}$ is exist in $\mathbb{C}[X, Y]$ such that

$$
Q(X(\xi), Y(\xi))=\sum_{i=0}^{m} a_{i}(X(\xi)) Y^{\dot{1}}(\xi)=0
$$

where $a_{m}(X) \neq 0$ and $i=0,1, \ldots, m$. By division theorem $\exists$ a polyn. $g(X)+h(X) Y$ such that

$$
\frac{d Q}{d \xi}=\frac{d Q}{d X} \frac{d X}{d \xi}+\frac{d Q}{d Y} \frac{d Y}{d \xi}=(g(X)+h(X) Y) \sum_{i=0}^{m} a_{i}(X) Y^{i}
$$

Assume that $m=1$ then coefficients of $Y^{\dot{1}}(i=$ $0,1)$ in eqn.(40), we get:

$$
\begin{gathered}
a_{1}(X)=a_{1}(X) \cdot h(X) \\
a_{0}^{\prime}(X)=a_{0}(X) \cdot h(X)+a_{1}(X) \cdot g(X) \\
a_{1}(X) \cdot\left(\frac{k-2 \sigma}{k} X-\frac{\beta}{3 k} X^{3}\right)=a_{0}(X) \cdot g(X) .
\end{gathered}
$$

Since $a_{i}(X)$ are polynomials, $a_{1}(X)$ is constant and $h(X)=0$ from eqn.(41)

Take $a_{1}(X)=1$ and for the equilibrium of $a_{0}(X)$ and $g(X), \operatorname{deg}(g(X))=1$.

Let $(X)=A_{0}+A_{1} X$, then

$$
a_{0}(X)=A_{0} X+\frac{1}{2} A_{1} X^{2}+B_{0}
$$

$A_{2}$ is integration constant. A nonlinear system of algebraic equations are obtained from $a_{0}(X), g(X)$ and eqn.(10).

$$
\begin{gathered}
A_{1}=\sqrt{\frac{-2 \beta}{3 k}}, B_{0}=\frac{k-2 \sigma}{k} \sqrt{\frac{3 k}{-2 \beta}}, A_{0}=0 \\
A_{1}=-\sqrt{\frac{-2 \beta}{3 k}}, B_{0}=-\frac{(k-2 \sigma)}{k} \sqrt{\frac{3 k}{-2 \beta}}, A_{0}=0
\end{gathered}
$$

under the conditions given by eqs. (12) and (13) in eq. (6), we have;

$$
Y(\xi)= \pm\left(\frac{k-2 \sigma}{k} \sqrt{\frac{3 k}{-2 \beta}}+\frac{1}{2} \sqrt{\frac{-2 \beta}{3 k}} X^{2}(\xi)\right)
$$

using eq.(14) and eq. (5), eqn.(14) is converted to following Ricatti equation:

$$
\left.U^{\prime}(\xi)= \pm\left(\frac{k-2 \sigma}{k} \sqrt{\frac{3 k}{-2 \beta}}+\frac{1}{2} \sqrt{\frac{-2 \beta}{3 k}} U^{2}(\xi)\right)\right)
$$

the solutions of the modified fractional Camassa Holm equation is obtained as:

$$
u_{1}(x, t)=\sqrt{\frac{3 k-6 \sigma}{\beta}} \tanh \left(\sqrt{\frac{2 \sigma-k}{2 k}}\left(x-k \frac{t^{\alpha}}{\Gamma(1+\alpha)}+\xi_{0}\right)\right)
$$




$$
\begin{aligned}
& u_{2}(x, t)=-\sqrt{\frac{3 k-6 \sigma}{\beta}} \tanh \left(\sqrt{\frac{2 \sigma-k}{2 k}}\left(x-k \frac{t^{\alpha}}{\Gamma(1+\alpha)}+\xi_{0}\right)\right) \\
& u_{3}(x, t)=\sqrt{\frac{3 k-6 \sigma}{\beta}} \operatorname{coth}\left(\sqrt{\frac{2 \sigma-k}{2 k}}\left(x-k \frac{t^{\alpha}}{\Gamma(1+\alpha)}+\xi_{0}\right)\right) \\
& u_{4}(x, t)=-\sqrt{\frac{3 k-6 \sigma}{\beta}} \operatorname{coth}\left(\sqrt{\frac{2 \sigma-k}{2 k}}\left(x-k \frac{t^{\alpha}}{\Gamma(1+\alpha)}+\xi_{0}\right)\right) \\
& u_{5}(x, t)=\sqrt{\frac{-3 k+6 \sigma}{\beta}} \tan \left(\sqrt{\frac{k-2 \sigma}{2 k}}\left(x-k \frac{t^{\alpha}}{\Gamma(1+\alpha)}+\xi_{0}\right)\right) \\
& u_{6}(x, t)=-\sqrt{\frac{-3 k+6 \sigma}{\beta}} \tan \left(\sqrt{\frac{k-2 \sigma}{2 k}}\left(x-k \frac{t^{\alpha}}{\Gamma(1+\alpha)}+\xi_{0}\right)\right) \\
& u_{7}(x, t)=\sqrt{\frac{-3 k+6 \sigma}{\beta}} \cot \left(\sqrt{\frac{k-2 \sigma}{2 k}}\left(x-k \frac{t^{\alpha}}{\Gamma(1+\alpha)}+\xi_{0}\right)\right) \\
& u_{8}(x, t)=-\sqrt{\frac{-3 k+6 \sigma}{\beta}} \cot \left(\sqrt{\frac{k-2 \sigma}{2 k}}\left(x-k \frac{t^{\alpha}}{\Gamma(1+\alpha)}+\xi_{0}\right)\right)
\end{aligned}
$$

\section{Functional Variable Method}

Introducing the wave transformation:

$$
u(x, t)=U(\xi), \xi=x-k \frac{t^{\alpha}}{\Gamma(1+\alpha)}
$$

Eqn. (1) reduce the form of eqn.(4) an ordinary differential equation:

$$
k U^{\prime \prime}(\xi)+(2 \sigma-k) U(\xi)+\frac{\beta}{3} U^{3}(\xi)=0
$$

according to functional variable method, we take $u_{\xi}=F(u)$ and some successive derivatives of $u(\xi)$ to following:

$$
\begin{gathered}
u_{\xi \xi}=\frac{1}{2}\left(F^{2}\right)^{\prime} \\
u_{\xi \xi \xi}=\frac{1}{2}\left(F^{2}\right)^{\prime \prime} \sqrt{F^{2}} \\
u_{\xi \xi \xi \xi}=\frac{1}{2}\left[\left(F^{2}\right)^{\prime \prime \prime} F^{2}+\frac{1}{2}\left(F^{2}\right)^{\prime \prime}\left(F^{2}\right)^{\prime}\right],
\end{gathered}
$$

Thus we obtain,

$$
\left(F^{2}\right)^{\prime}=\frac{2(k-2 \sigma)}{k} u-\frac{2 \beta}{3 k} u^{3}
$$

integrating the eqn. (25), we have

$$
F(u)=\sqrt{-\frac{\beta}{6 k}} u \sqrt{u^{2}-\frac{6(k-2 \sigma)}{\beta}}
$$

from $u_{\xi}=F(u)$ and (26) we deduce that

$$
\int \frac{d u}{u \sqrt{u^{2}-\frac{6(k-2 \sigma)}{\beta}}}=\sqrt{-\frac{\beta}{6 k}}\left(\xi+\xi_{0}\right)
$$

$\xi_{0}$ is a integration constant. After integrating (27), we achieve the following exact solutions:

Case 1. If $k=2 \sigma$, then

$$
u_{1}(x, t)= \pm \frac{1}{\sqrt{-\frac{\beta}{6 k}}\left(x-k \frac{t^{\alpha}}{\Gamma(1+\alpha)}+\xi_{0}\right)}
$$

Case 2. If $\frac{6(k-2 \sigma)}{\beta}>0$, then 


$$
\begin{aligned}
& u_{9}(x, t)=\sqrt{\frac{6(k-2 \sigma)}{\beta}} \sec \left(\sqrt{\frac{2 \sigma-k}{k}}\left(x-k \frac{t^{\alpha}}{\Gamma(1+\alpha)}+\xi_{0}\right)\right) \\
& u_{10}(x, t)=-\sqrt{\frac{6(k-2 \sigma)}{\beta}} \sec \left(\sqrt{\frac{2 \sigma-k}{k}}\left(x-k \frac{t^{\alpha}}{\Gamma(1+\alpha)}+\xi_{0}\right)\right) \\
& u_{11}(x, t)=\sqrt{\frac{6(k-2 \sigma)}{\beta}} \csc \left(\sqrt{\frac{2 \sigma-k}{k}}\left(x-k \frac{t^{\alpha}}{\Gamma(1+\alpha)}+\xi_{0}\right)\right) \\
& u_{12}(x, t)=-\sqrt{\frac{6(k-2 \sigma)}{\beta}} \csc \left(\sqrt{\frac{2 \sigma-k}{k}}\left(x-k \frac{t^{\alpha}}{\Gamma(1+\alpha)}+\xi_{0}\right)\right)
\end{aligned}
$$

Case 3. If $\frac{6(k-2 \sigma)}{\beta}<0$, then

$$
\begin{aligned}
& u_{13}(x, t)=\sqrt{\frac{6(2 \sigma-k)}{\beta}} \operatorname{sech}\left(\sqrt{\frac{k-2 \sigma}{k}}\left(x-k \frac{t^{\alpha}}{\Gamma(1+\alpha)}+\xi_{0}\right)\right) \\
& u_{14}(x, t)=-\sqrt{\frac{6(2 \sigma-k)}{\beta}} \operatorname{sech}\left(\sqrt{\frac{k-2 \sigma}{k}}\left(x-k \frac{t^{\alpha}}{\Gamma(1+\alpha)}+\xi_{0}\right)\right) \\
& u_{15}(x, t)=\sqrt{\frac{6(2 \sigma-k)}{\beta}} \operatorname{csch}\left(\sqrt{\frac{k-2 \sigma}{k}}\left(x-k \frac{t^{\alpha}}{\Gamma(1+\alpha)}+\xi_{0}\right)\right) \\
& u_{16}(x, t)=-\sqrt{\frac{6(2 \sigma-k)}{\beta}} \operatorname{csch}\left(\sqrt{\frac{k-2 \sigma}{k}}\left(x-k \frac{t^{\alpha}}{\Gamma(1+\alpha)}+\xi_{0}\right)\right)
\end{aligned}
$$

\section{Discussion}
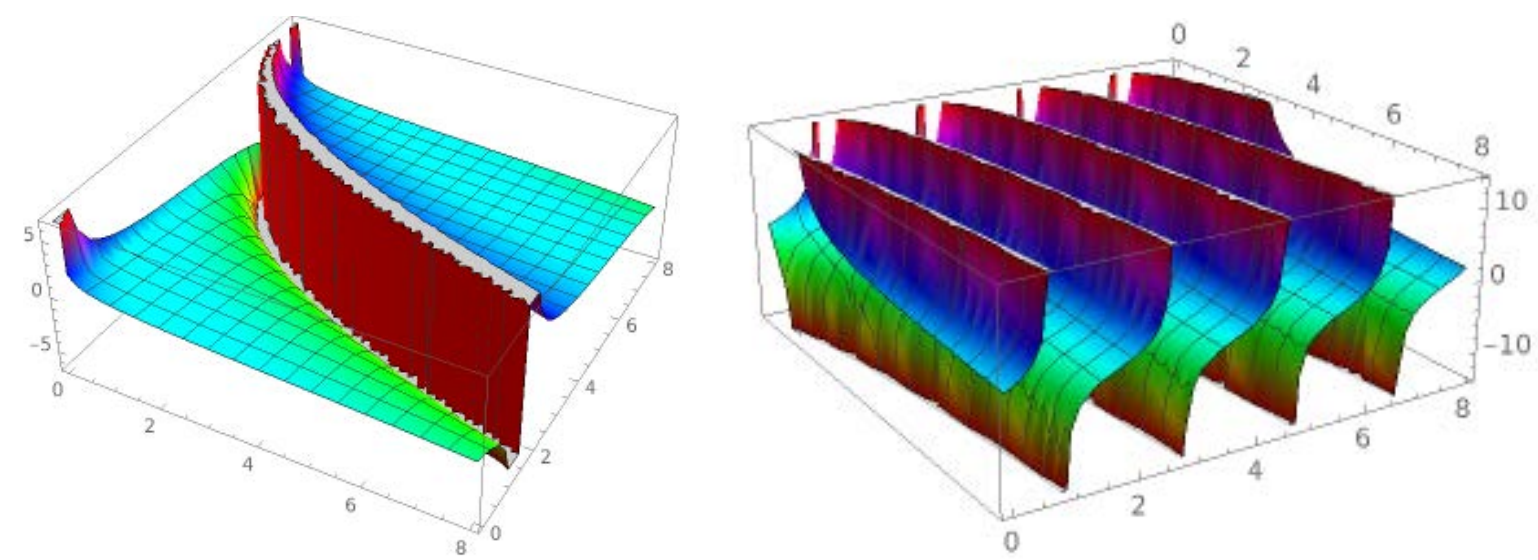

Figure $1-3 \mathrm{D}$ plots of the exact solution $u_{3}(x, t)$ and $u_{5}(x, t)$ respectively, for the case of; $k=-2.23, \sigma=-0.75, \beta=2.07, \alpha=0.41$ and $k=0.85, \sigma=-1.81, \beta=-2.88, \alpha=0.3$ 

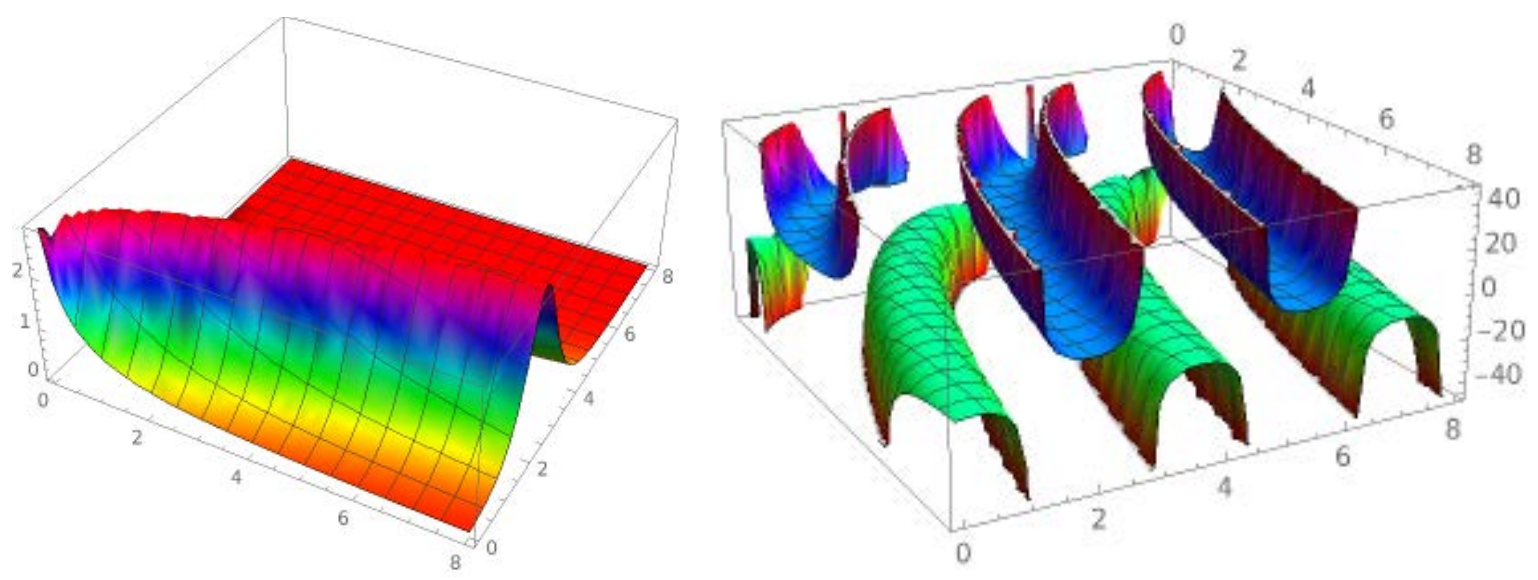

Figure $2-3 \mathrm{D}$ plots of the exact solution $u_{9}(x, t)$ and $u_{12}(x, t)$ respectively, for the case of; $k=1.009, \sigma=-1.17, \beta=2.39, \alpha=0.399$ and $k=-1.55, \sigma=-3.4, \beta=0.74, \alpha=0.4$
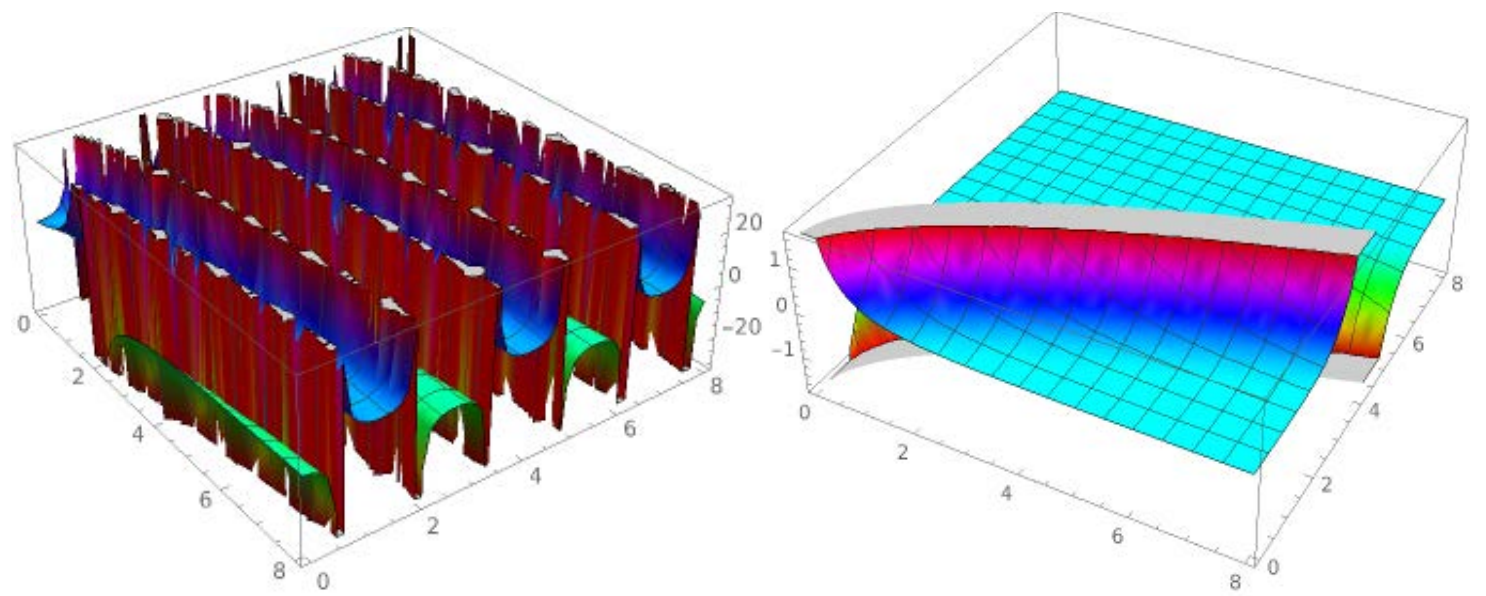

Figure 3 - 3D plots of the exact solution $u_{13}(x, t)$ and $u_{16}(x, t)$ respectively, for the case of; $k=0.48, \sigma=1.43, \beta=2.39, \alpha=0.44$ and $k=1.27, \sigma=-1.86, \beta=-2.08, \alpha=0.54$

In this section, we have presented some comparisons about physical behaviours. First method gives us hyperbolic solutions and trigonometric solutions (see Fig. 1-4); on the other hand, the second one gives rational solutions, hyperbolic solutions and periodic solutions. Compacton waves and kink soliton waves have been achieved via first method, compacton waves and bell-shaped soliton waves have also been obtained via second method. Thanks to these comparisons, we can have an idea about physical interpretation of other nonlinear FPDEs.

\section{Conclusion}

In this study, thanks to FVM and FIM, we have attained new exact solutions of the fractional modified Camassa - Holm equation. The proposed methods are powerful, reliable and effective to obtain nonlinear FPDEs defined by conformable derivative. Main advantage of these methods is generating more type of solution functions than the other analytical methods. Trueness of solution functions have also been proved by using the Mathematica program. Additionally, some 3D graphs have been examined for proper values of the parameters. Obtained results and comparisons show that methods will be useful to make the physical interpretation of nonlinear FPDEs arising in applied physics and mathematics.

\section{References}

1 Podlubny, Fractional Differential Equations: an Introduction to Fractional Derivatives, Fractional Differential Equations, to Methods of Their Solution and Some of Their Applications. Elsevier, 1998K. 
Satou, H. Ikezoe, S. Mitsuoka, K. Nishio, and S. C. Jeong, Phys. Rev. C 65 (2002) 054602.

2 Hilfer, R., Applications of fractional Calculus in Physics, World Scientific Publishing. River Edge, NJ, USA (2000).

3 Kilbas, A., Srivastava, H. M., Trujillo, J. J., Theory and Applications of Fractional Differential Equations. North-Holland Mathematics Studies, Elsevier Science, Amsterdam, The Netherlands, 204 (2006).

4 Caputo, M., Elasticita e Dissipazione. Zanichelli, Bologna, (1969).

5 Li, C., Guo, Q., Zhao, M., On the solutions of (2+1)-dimensional time - fractional Schrödinger equation, Appl. Math. Lett., 94 (2019): 238-243.

6 Atangana A., D. Baleanu, D., New fractional derivatives with nonlocal and non-singular kernel: theory and application to heat transfer model, Therm. Sci., 20 (2016): 763.

7 Liu, W., Chen, K., The functional variable method for finding exact solutions of some nonlinear time fractional differential equations, Pramana $-\mathrm{J}$. Phys., 81 (2013): 3.

8 Wazwaz, A. M.,The extended tanh method for new solitons solutions for many forms of the fifth order KdV equations, Appl. Math. Comput., 184 (2), (2007): 1002-1014.

9 Hosseini, K., Ansari, R., Gholamin, P., Exact solutions of some nonlinear systems of partial differential equations by using the first integral method, J. Math. Anal. and Apps., 387, (2012): 807 -814 .

10 Rezazadeh, H., et al., New exact solutions of nonlinear conformable time-fractional Phi-4 equation. Chinese Journal of Physics, 56(6) (2018) 2805-2816.

11 Kaplan, M., Akbulut, A,. Bekir, A., Solving space-time fractional differential equations by using

12 modified simple equation method, Commun. Theor. Phys., 65(5) (2016): 563.

13 Odabasi, M., Traveling wave solutions of conformable time-fractional Zakharov-Kuznetsov and Zoomeron equations, Chinese Journal of Physics, 64 (2020): 194-202.

14 Bekir, A., Guner, O., Bhrawy, A. H., Biswas, A., Solving nonlinear fractional differential equations using exp-function and G'/G-expansion methods. Rom. J. Phys. 60(3-4) (2015): 360-378.

15 Pandir, Y., Gurefe Y., Misirli, E., The extended trial equation method for some timefractional differential equations, Discrete Dyn. Nat. Soc., 2013 (2013): 491359.

16 Gurefe, Y., The generalized Kudryashov method for the nonlinear fractional partial differential equations with the beta-derivative, Revista Mexicana de Física, 66 (2020): 771-781.

17 Youssri, Y. H., Abd-Elhameed, W. M., Romanian Journal of Physics 63 (2018): 107.

18 Öztürk, Z., Bilgil, H., Exact Solutions of Rosenzweig-Macarthur (RM) Model Equations by Using Exp Function Method. Avrupa Bilim ve Teknoloji Dergisi, 16 (2019): 427-433.

19 Kurt, A., Tasbozan, and O., Cenesiz, Y., Homotopy analysis method for conformable burgers korteweg-de vries equation, Bull. Math. Sci. Appl., 17 (17) (2016): 23.

$20 \mathrm{He}$, J. H., Variation iteration method-a kind of non-linear analytic technique: some examples, Int. J. Non-linear Mech. 34 (4) (1999): 699-708.

21 Prakash, A., Kumar, M., Baleanu, D., A new iterative technique for a fractional model of nonlinear Zakharov-Kuznetsov equations via Sumudu transform, Appl. Math. Comput., 334 (2018): 30-40.

22 Akbulut, A., Kaplan, M., Auxiliary equation method for time-fractional differential equations with conformable derivative, Comput Math Appl., 75 (2018) 876-882.

23 Guner, O., New exact solutions to the spacetime fractional nonlinear wave equation obtained by the ansatz and functional variable methods, Opt Quant Electron, 50 (2018): 38.

24 Kadomtsev, B. B., Petviashvili, V. I., Relativistically invariant two-dimensional models of field theory which are integrable by means of the inverse scattering problem method, Sov. Phys. JETP.,74 (6) (1978): 1017-1027.

25 Cheng, Y. F., Dai, T. Q., Exact solutions of the Klein-Gordon equation with a ring-shaped modified Kratzer potential. Chin. J. Phys., 45 (5) (2007): 480-487.

26 Khater, M. M. A., Zahran, E. H. M., New method to evaluating exact and traveling wave solutions for non-linear evolution equations, J. Comput. Theor. Nanosci, 12 (2015): 1 - 9.

27 Eslami, M., Rezazadeh, H., The first integral method for $\mathrm{Wu}$-Zhang system with conformable time fractional derivative, Calcolo, 53 (2016): 475-485.

28 Inc, M.,. Inan, E., Ugurlu, Y., New applications of the functional variable method. Optik, 136 (2017): 374- 381.

29 Zulfiqar, A., Ahmad, J., Exact solitary wave solutions of fractional modified Camassa-Holm equation using an efficient method, Alexandria Engineering Journal, 59(5) (2020): 3565-3574.

30 Khalil R., Al Horani, M., Yousef, A., Sababheh, M., A new definition of fractional derivative, Journal of Computational and Applied Mathematics, 264 (2014): 65-70. 